\title{
CAN THE FUNDAMENTAL SYSTEM BE REPLACED BY A RE FERENCE SYSTEM OF GALAXIES
}

\author{
Walter Fricke \\ Astronomisches Rechen-Institut, Heidelberg
}

1. Purpose of a Fundamental System of Positions and Proper Motions.

Quasistellar objects with and without radio emission have recently given unforeseen and vigorous impetus to astrometry. Great effort has been made in the search for the optical counterparts of radio sources. For the purpose of identifications accurate positions were demanded, optical as well as those by radio techniques. Optical positions of radio sources are also needed to serve as calibrators for radio positions. In all cases the objects under consideration are optically much fainter than those ever used previously in high precision astrometry. Finally, methods have been developed for the absolute measurement of radio positions to an accuracy which competes with that known from fundamental observations of bright stars.

In view of forthcoming possibilities of establishing a frame of reference of radio sources by absolute observations of radio positions, it appears appropriate to ask whether the traditional fundamental system of steller positions and proper motions can be replaced by a reference system of galaxies or extragalactic radio sources.

The conventional fundamental system in current use is represented by the positions and proper motions of 1535 stars brighter than visual magnitude 7.5 and fairly uniformly distributed over the whole sky. It was compiled from absolute observations of stellar positions at various epochs over a period of about one hundred years. The term "absolute" means that the observations have been carried out independently of measurements of other stellar positions. The fundamental system is given by the data of the Fourth Fundamental Catalogue (FK4). Its purpose is to serve as the reference system of positional astronomy. It is to fulfill the requirements of a primary frame of reference for the determination of the motions of the stars and the bodies of the planetary system. It should represent the inertial frame of reference.

In fulfillment of its task as the primary frame of reference the FK4 served to establish secondary reference frames of stellar positions in both hemispheres, established by means of differential observations with transit circles. In this way positions of a great number of stars down to about visual magnitude 9.5 have been determined in the system of FK4 (about 24,000 stars of the northern sky within the AGK3R program and about the same number in the southern sky in the SRS program). The stars in AGK3R 
served as reference stars for the measurement of photographic positions and for the formation of the AGK3. The SRS stars will play a similar role in the southern sky.

In principle, the formation of reference frames of higher order and with fainter stars is possible as far as the accuracy requirements permit. Murray et al. (1969) have recently determined photographic positions in the FK4 system of a number of radio sources of the $3 \mathrm{C}$ Catalogue. For this purpose, first the positions of a number of $\mathrm{BD}$ stars were referred to the FK 4 by means of differential observations with a transit circle. The BD stars then provided the reference system for the photographic determination of positions of fainter stars $\left(m_{p q} \approx 13\right)$, and the photographic images of the radio sources were measured ${ }^{\text {th }}$ th respect to the latter. By this technique objects of visual magnitude eighteen and fainter were reached. The work indicates the way in which extragalactic quasistellar objects can be tied to the traditional fundamental system.

2. Limitations of the Fundamental System and possible Improvements.

The limitation of the fundamental system to bright stars was caused by the optical limit of older transit circles and by systematic errors depending on the magnitude of the stars. The extension of the system down to the magnitude limit of about 9.0 is one of the desiderata for FK5. While there is some hope that in the northern sky the systematic errors depending on the magnitude can be kept under control down to 9th magnitude, it appears doubtful that the same is true for the southern hemisphere. No doubt, modern observations in north and south take all necessary precautions to avoid errors depending on magnitude; but old observations, which could be assumed to be free from magnitude effects and which could provide proper motions free from errors depending on the magnitude, are not available for the whole sky.

Here we encounter one of the main problems in the establishment of a frame of reference, namely, its uniformity over a large range of magnitudes. We need a system that is identical for all objects, irrespective of their brightness.

A trivial limitation of the fundamental system is, of course, the accuracy of observations which contributed to the formation of the system. In the past decades standard errors of single observations with transit circles were reported between $\pm 0 ! 30$ and $\pm 0 ! 45$ in each coordinate. At present, the accuracy can be higher. At Brørfelde Observatory of the University of Copenhagen stars in the visual magnitude range from 3.0 to 11.0 were measured in the system of FK4. These observations are among the most accurate made so far; the standard errors of single observations in right 
ascension and declination are

$$
\varepsilon_{\alpha} \cos \delta= \pm 0.0152, \quad \varepsilon_{\delta}= \pm 0 ! 219 \text {. }
$$

The same high accuracy - or even a little better - has been reported for the transit circle observations presently being carried out at Perth, West Australia, by an expedition of the Hamburg Observatory.

In FK4, the standard errors describing the internal accuracy of positions differ from star to star depending on the number and quality of observations. They are of the order of

$$
\varepsilon_{\alpha} \cos \delta \simeq \pm 0.003, \quad \varepsilon_{\delta} \simeq \pm 0 ! 04,
$$

except in the polar regions, where they are greater. These values hold for the mean epoch of observations, which differ for each star. The mean epoch of the $\alpha$ - system is 1935, and it is 1925 for the $\delta$ - system. The standard errors describing the systematic accuracy differ for various zones of the sky. At mean epoch they are of the order of

$$
\varepsilon_{\alpha} \cos \delta \simeq \pm 0.002, \quad \varepsilon_{\delta} \simeq \pm 0: 016,
$$

except for the southern polar region, where they are greater.

In FK4, the averaged standard errors describing the internal accuracy of centennial proper motions are

$$
\varepsilon_{\mu_{\alpha}}= \pm 0 ! 16, \quad \varepsilon_{\mu_{\delta}}= \pm 0 ! 17 \text { per century }
$$

and the averaged standard errors describing the systematic accuracy are

$$
\varepsilon_{\mu_{\alpha}}= \pm 0 ! 17, \quad \varepsilon_{\mu_{\delta}}= \pm 0 ! 07 \text { per century. }
$$

These numbers represent linear averages over the whole sky. The average values resulting from the combination of internal and systematic accuracy are

$$
\varepsilon_{\mu_{\alpha}}= \pm 0 ! 24, \quad \varepsilon_{\mu_{\delta}}= \pm 0 ! 18 \text { per century. }
$$

For fundamental systems preceding the FK4 corresponding values are not known. It is certain, that the average accuracy has been increasing only slowly, and it appears that the increase will also be slow in future.

Even more important than the average increase of accuracy appears to be the elimination of inhomogeneities of the system. At present, the systematic errors differ too much for different parts of the sky or even from one area 
to the other. This situation becomes apparent in a comparison between the systems of FK4 and N30, the overall accuracy of which cannot be very different. Whatever quantities of the two systems are compared, in all cases the deviations of the two systems from each other show regional structure. As an example, Figure 1 shows the distribution of differences $\Delta \mu \delta$ (FK 4 N30) over the sky according to Brosche, Nowacki and Strobel (1964). Different shading indicates regions with different centennial values in the range

$$
-0: " 80<\Delta \mu_{\delta}<+0: ! 60 .
$$

There dominate connected areas in which $\Delta \mu_{\delta}$ takes either positive values smaller than $+0 ! 20$ or negative values greater than $-0 ! 20$. Compared to these the areas with large deviations are satisfactorily small. For a full appreciation of the information in Figure 1, the effects on determinations of galactic rotation and lunisolar precession must be considered. The latter are essentially determined by $\mu_{\delta}$, since their contributions to $\mu_{\alpha}$ are much smaller. Dort's galactic constant B is of the order of $-0: 20$ per century and the precessional correction $\Delta \mathrm{n}$ of the order of $+0 ! 40$. Determinations of these quantities carried out separately in the systems of FK4 and N 30 give results which are in excellent agreement, if proper motions of stars distributed over the whole sky are used. The agreement is much less satisfactory, if only parts of the sky are used. From Figure 1 it is obvious that determinations based, for example, on the zones from $-20^{\circ}$ to $+20^{\circ}$ and from $+20^{\circ}$ to $+60^{\circ}$ must differ appreciably in their results. Along the galactic equator it is also apparent that results from the northern and southern hemisphere would differ owing to systematic errors.

Finally, there is the deficiency of the present fundamental system arising from erroneous values of precession and an erroneous motion of the equinox. While these errors do not diminish the utility of FK4 as a frame of reference for some purposes, they must be eliminated from the proper motions in all investigations on stellar motions. Whenever the user needs to know motions referred to the local inertial frame of reference, he must apply corrections to the proper motions of FK4 and to all those which are referred to FK4. All proper motions $\mu_{\alpha}, \mu_{\delta}$ given in catalogues have to be corrected as follows

$$
\begin{aligned}
& \left(\mu_{\alpha}\right) \cos =\mu_{\alpha}-\Delta \mu_{\alpha} \\
& \left(\mu_{\delta}^{\alpha}\right) \cos =\mu_{\delta}-\Delta \mu_{\delta}^{\alpha}
\end{aligned}
$$

where, in the usual notation,

$$
\begin{aligned}
& \Delta \mu_{\alpha}=\Delta \mathbf{k}+\Delta \mathrm{n} \sin \alpha \tan \delta, \\
& \Delta \mu_{\delta}^{\alpha}=\Delta \mathrm{n} \cos \alpha \text {, } \\
& \begin{array}{l}
\Delta \mathrm{k}=\Delta \mathrm{m}-\Delta \mathrm{e}, \\
\Delta \mathrm{m}=\Delta \mathrm{p}_{1} \cos \mathcal{E}-\Delta \boldsymbol{\lambda}, \\
\Delta \mathrm{n}=\Delta \mathrm{p}_{1} \sin \mathcal{E} .
\end{array}
\end{aligned}
$$




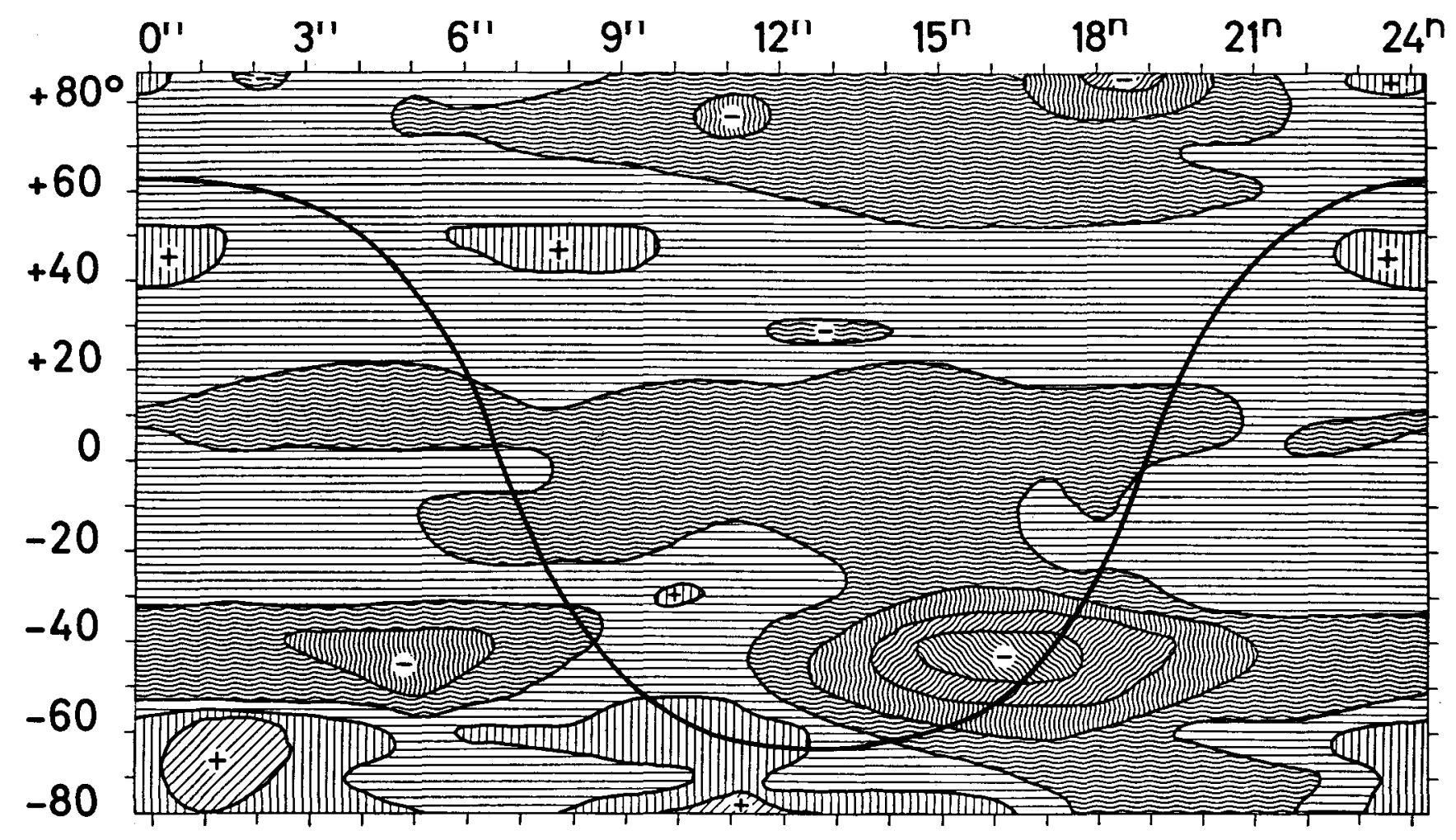

$\Delta \mu_{\delta}(F K 4-N 30$ for 1950$)$, cent. unit $0 . .01$

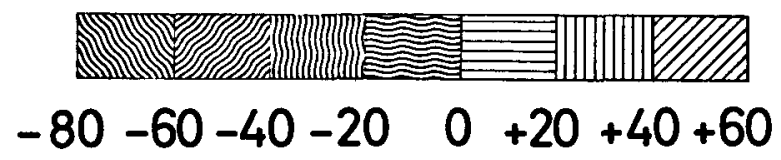

FIGURE 1 
$\mathcal{E}$ denotes the obliquity of the ecliptic. From recent determinations carried out by Fricke $(1967,1968)$ the recommended values of the corrections are

$$
\begin{aligned}
\Delta \mathrm{n} & =+0 ! 44 \text { corresponding to } \Delta \mathrm{p}_{1}=+1 ! ' 10 \text { per century, } \\
\Delta \lambda+\Delta e & =+1 ! 20 \text { per century. }
\end{aligned}
$$

$\Delta \mathrm{p}_{1}$ and $\Delta \boldsymbol{\lambda}+\Delta e$ are accurate to about \pm 0 !" 15 per century.

In summarizing we may draw the following conclusions for future work on the fundamental system: Observations available at the present time suggest the extension of the fundamental system as the primary frame of reference down to the magnitude limit of about 9.0 visual. This means a considerable increase of the number of fundamental stars. The number of suitable stars in the whole sky will, however, scarcely exceed about 5000 objects (compared to 1535 fundamental stars at present ); suitability may be decided by the number of observations and their systematic and individual accuracy.

\section{Proper Motions with Respect to Galaxies.}

Although the utility of galaxies as a reference frame was already recognized by Herschel (1785) and Laplace (1797), programs for measuring proper motions of stars with respect to galaxies were initiated only about twenty years ago. Since Wright (1950) and Deutsch (1954) made detailed proposals, considerable progress has been made in the development of techniques, in experience with measurements, and through the discovery of extragalactic radio sources and their connection with quasistellar objects.

The original idea was to determine positions of galaxies such that they might provide a frame of reference for stellar motions. Among others it was Kopff (1936) who gave arguments in favor of this idea in the very same year that he completed the fundamental catalogue FK3 produced by traditional methods. Wright also advocated the construction of a system of positions of galaxies. Since the experience of Vasilevskis $(1954,1957,1963)$ with Lick astrographic plates and of the Pulkovo astronomers with the measurability of plates taken with the normal astrograph, more emphasis has been given to the idea of measuring high precision stellar proper motions strictly differential to galaxies than to measuring accurate positions.

The measurements yield proper motions which are free of errors arising from incorrect precession and from errors in the motion of the equinox. Preliminary results of the determination of motions referred to galaxies have been reported by Fatchikhin (1968). Some basis is therefore available for evaluating what has been achieved already and what may be gained by the method in future. 
First, it has been obvious from the beginning that proper motions alone cannot replace the fundamental system, since there are important tasks which require the knowledge of positions. Precise positions are needed for identifying extragalactic objects and for providing the reference frame for the measurement of positions of other objects of all kinds, extragalactic, galactic, and planetary.

Precise absolute proper motions determined by differential measurements with respect to galaxies may, however, be extremely useful for (a) the determination of precession and of systematic errors in fundamental proper motions, (b) investigations of galactic rotation, (c) investigations of the velocity distribution of stars.

Fatchikhin reported the determination of precessional corrections from absolute proper motion $\mu_{\mathrm{Gal}}$ of 742 AGK3 stars which were measured differentially with respect to galaxies on plates (epoch difference of about 22 years) taken with the Pulkovo normal astrograph. For these stars proper motions $\mu_{\mathrm{AGK} 3}$ are known which are in the system of FK4. From the differences

$$
\Delta \mu=\mu_{\mathrm{AGK} 3}{ }^{-\mu_{\mathrm{Gal}}}
$$

Fatchikhin found the correction

$$
\Delta \mathrm{p}_{1}=+1 ! " 11 \pm 0 ! 11
$$

which is in perfect agreement with the value determined by Fricke (1967) on the basis of FK4 proper motions. Fatchikhin's value for

$$
\begin{aligned}
\Delta E= & \Delta \lambda+\Delta e, \text { namely, } \\
& \Delta \lambda+\Delta e=+0 ! 49 \pm 0 ! 04
\end{aligned}
$$

is smaller than the value derived by Fricke and others by a factor of more than two. While Fatchikhin considers the difference to be real, it appears that the results for $\Delta \mathrm{p}_{1}$ and $\Delta \lambda+\Delta e$ are better than could be expected. The accuracy of this determination rests upon the individual and systematic accuracy of the AGK 3 in the respective regions and on the measurements with respect to galaxies on the Pulkovo plates. For the AGK3 motions an accuracy of about one second of arc per century can be claimed. For differential motions referred to images of galaxies and based on an epoch interval of 22 years the accuracy can be estimated to be at highest $\pm 1 ! 45$ per century. Included in the estimated error of the AGK3 motions are the systematic errors of the FK4, which must be expected to show regional structure. In referring to the distribution of regional differences between FK4 and N30 shown in Figure 1, and to the experience with regional solutions for precessional corrections from FK4 proper motions, Fatchikhin's result 
for $\Delta p_{1}$ seems to be a felicitous accident, and the result of $\Delta E$ seems to be affected by some systematic errors which are not unexpected. These considerations suggest that attempts to determine precession from cosmic proper motions - those referred to galaxies - must not be expected to provide reliable results unless such proper motions cannot be compared with improved fundamental ones, or unless cosmic proper motions are available over the whole sky.

The situation is similar with determinations of galactic rotation, which suffer from the fact that no direct measurements of motions with respect to galaxies are possible in the zone of avoidance. There remains the advantage of cosmic motions for studies on the velocity distribution of faint stars. One may wonder whether for this purpose the traditional and tedious methods of high precision astrometry are appropriate, even if modern facilities of measuring and data processing are applicable. It appears that an entirely unconventional instrument for astrometric purposes, the 48-inch Palomar Schmidt, has provided excellent material for an enormous proper motion survey of faint stars carried out by Luyten during the past years. The great advantages of Luyten's techniques of blinking and measuring the plates have been illustrated by many important discoveries.

These comments on the limitations of high precision proper motions referred to galaxies may not be understood as a discouragement of further effort. Programs like those at Lick and at Pulkovo Observatory are urgently necessary in many respects. For this very reason they need to be protected against unqualified expectations and hopes that they very probably will not be able to fulfill. These critical remarks appear to be necessary at a time when from elementary courses almost every student of astronomy expects that the Lick plates will provide the inertial frame of reference for all purposes of interest.

\section{Positions of Galaxies and Radio Sources.}

The structure of images of galaxies and radio sources plays an important role in the definition of positions of such objects. The measurability of a position may differ from one instrument to another and depends on the frequency of radiation and other properties of the objects of measurement. It has been known for a long time that among faint extragalactic objects many exist with stellar-like images. These objects would be suited for measurements of positions, but because of their faintness there was not much interest in them. This situation changed radically after a number of radio sources had been identified with quasistellar objects. For the purpose of identification precise measurements of optical and radio positions have become necessary. Optical positions also have to provide a frame of reference for calibrating measurements of positions with radio telescopes. 
For example, plates taken with the 48-inch Palomar Schmidt were used by Bolton (1968) for the measurement of 78 optically identified radio sources. Yale catalogue stars near each radio source served as reference stars. Bolton achieved in this way positions to an accuracy of less than one second of arc in each coordinate. The highest accuracy ever reached in measuring positions of objects fainter than magnitude 18, was reported by Murray et al. (1969). They determined optical positions of sixteen radio sources in the system of FK4. First, they used a transit circle for measuring positions of BD stars with respect to FK4; second, they used plates of an astrographic telescope to refer positions of reference stars of about magnitude 13 to the BD stars. Finally the positions of the radio sources were measured relative to these reference stars on plates taken at Greenwich Observatory either with the 26-inch refractor or the Isaac Newton Telescope. Their standard errors for the position of a radio source obtained from at least two plates are of the order of

$$
\mathcal{E}_{\alpha}= \pm 0 ! 25, \quad \mathcal{E}_{\delta}=+0 ! 12 .
$$

If other measurements will confirm this very high accuracy, there appears to be no technical obstacle any more against the construction of a fundamental system extending from brightest stars to faint quasistellar objects.

For the determination of radio positions various methods have been used: (a) With radio interferometers positions have been determined with an accuracy better than one second of arc. The system has been provided by observing radio sources associated with optical objects with known positions.

(b) The method of lunar occultations has given accurate positions - with errors of the order of one second of arc or less - for compact radio sources. (c) Of greatest interest for constructing a fundamental system are, however, the methods for determining absolute radio positions as described and applied by Elsmore and Mackay (1969), who measured compact radio sources with the one-mile radio telescope in Cambridge, England. These positions have been obtained with an accuracy of about $\pm 1^{\prime \prime}$ in right ascension and $\pm 2^{\prime \prime} \operatorname{cosec} \delta$ in declination. Such observations which are independent of measurements of optical positions may help to decide whether real and vitally important differences exist between radio and optical positions. Such differences cannot yet be excluded even for the most compact radio sources.

There are indications that the accuracy of absolute radio positions will soon be better than one second of arc, and that compact sources will provide a frame of references which could soon become as accurate as the fundamental system of positions. Such possibilities suggest effort in the direction of a fundamental frame of reference comprising positions and proper motions of stars from the brightest to faint ones and positions of compact galaxies and extragalactic radio sources in a uniform system. 


\section{DISCUSSION}

Dieckvoss: Is it possible to pour some water in your wine, because if you get such good determinations when you have only altitude and azimuth which you transform into right ascension and declination, then, in that reduction a number of errors are introduced. Fricke uses an equation there, differential with respect to the FK4. If it has the accuracy that we think, then we have tied those positions into FK 4 .

Fricke: I would make it plain here that the accuracy quoted is that of the position at the mean epoch. And if this quantitiy holds for, say 1925, then now, almost fifty years later the accuracy is much less because of the errors of the proper motions.

Murray: In this connection I would like to mention a program that we have started at Herstmonceux, and I would very much like to see other people try it. This is to photograph with long focus telescopes using parallaxmagnitude reduction methods of rotating sectors, the FK4 stars and to try to determine their right ascensions and declinations with respect to the AGK3 stars around them. This gives a sort of feed back between the FK4 system, through our new secondary system that Dr. Dieckvoss has very kindly provided us with, and to check the agreement. I have no results to show you, but I think that this is an easy program to carry out. And I would like to suggest that other people try it as well. You have only spot checks, but there are a lot of FK4 stars that can be done in this way.

Fricke: May I make one additional comment on this problem; that I personally am convinced that for the measurement of the positions of the

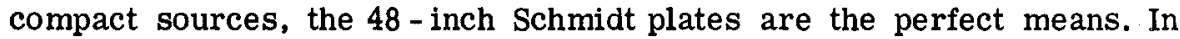
such a small field as Luyten said this will provide us the best and most accurate positions for these compact sources. Would you agree?

Murray: Yes, in fact I am not even convinced it is necessary to do the step method. I have - perhaps I should not quote, because this is only a secondhand communication - a letter forwarded to me from someone at Mount Wilson saying that some work there on determining positions of radio sources directly from three stars produced the sort of accuracies I claimed there. And that is a direct step. But it was not a communication to me so I will not mention any names.

Fricke: I personally would always like to leave the AGK3 out when getting down to faint objects, because, in my opinion, that is, not for the future, but for the past. The accuracies get better, you see, with time.

Strand: Would Dr. Fricke like to comment on this accuracy claimed by Oort's group of 0!'2 with a radio telescope. 
Fricke: These are measurements that have just been made at the present time, so I can only guess that perhaps they measure the zenith distance without any reference to optical positions, without any radio calibrator, but with a method similar to that published by McKay, and somebody else in the Monthly Notices.

Eichhorn: Is it not impossible to determine the motion of the equinox from declination observations?

Fricke: One cannot, of course. You see I would not yet ask to measure the position of the sun with respect to radio sources, but we may well find very soon that that is also possible.

Eichhorn: I understood you to say that from absolute declination proper motions there is found also the motion of the equinox, 0:"7 per century. Is this what you say?

Fricke: No, no. You see the correction to the motion of the equinox, $\Delta \mathrm{e}$, is the sum of the corrections, then the star has position less $\Delta \mathrm{e}$. And that one can measure by comparing the AGK3 proper motions in right ascension with the galactic term, with the proper motions with respect to the galaxy.

Murray: Can I just make a short comment about the radio positions. There are outstanding large discrepancies between the radio and optical positions in right ascension. These may be due to the methods used for the reduction of the radio observations. I was talking to McKay only last week and they suspect now that there may be something that will explain discrepancies at high declinations between our positions and theirs.

Fricke: I can tell you it is an unfortunate thing because for the Leyden observations, we at Heidelberg are still searching for methods to reduce them rigorously. As some of you well know we get into trouble near the pole.

Murray: I think the trouble is that there are very few radio sources at high declinations. Whether it is the cosmical effect or not I do not know. There is only one that we have determined, I think, at about $70^{\circ}$ declination and this stands out. The question is, then, is this a misidentification or is it in the radio reductions.

Fricke: Of course, the optical positions and the radio positions may not be identical. 